\title{
ANÁLISE DO CONTÁGIO ENTRE OS MERCADOS BOLSISTAS INTERNACIONAIS NO ÂMBITO DA CRISE FINANCEIRA GLOBAL
}

ANALYSIS OF THE CONTAGION AMONG INTERNATIONAL STOCK MARKETS IN THE CONTEXT OF THE GLOBAL FINANCIAL CRISIS ANÁLISIS DEL CONTAGIO ENTRE LOS MERCADOS BOLSISTAS INTERNACIONALES EN EL ÁMBITO DE LA CRISIS FINANCIERA GLOBAL

\author{
Vitor Manuel de Sousa Gabriel* \\ José Ramos Pires Manso**
}

\section{RESUMO:}

Neste trabalho é estudado o impacto da crise financeira global ao nível do contágio entre os mercados bolsistas. Com este objetivo, foram selecionados doze dos maiores mercados bolsistas, europeus e não europeus, e foi escolhido o período compreendido entre 4/10/1999 e 30/06/2011. Para identificar a ocorrência de efeito de contágio, recorreu-se ao modelo exponencial de heterocedasticidade condicionada (EGARCH) e a testes aos coeficientes de correlação, de modo a perceber se os coeficientes registados no subperíodo Crise Financeira Global diferem dos registados nos subperíodos anteriores. As conclusões obtidas revelam que os coeficientes de correlação sofreram um aumento significativo no último subperíodo, o que confirma a existência de efeitos de contágio entre os mercados bolsistas estudados.

Palavras-chave: Crise financeira global, mercados bolsistas internacionais, EGARCH, efeito de contágio.

\begin{abstract}
:
This paper examines the impact of the global financial crisis in terms of contagion between stock markets. To this end, twelve of the largest European and non-European stock exchange markets were selected, and the period from 10/04/1999 to 30/06/2011was chosen. So that we could identify the occurrence of the contagion effect, we used the exponential generalized autoregressive conditional heteroskedasticity (EGARCH) model. In order to understand if the coefficients recorded in the Global Financial Crisis sub period differ from those recorded in previous sub-periods, we used correlation coefficient tests. The obtained findings reveal that the correlation coefficients increased significantly in the last sub-period, confirming the existence of contagion effects among the studied stock markets.
\end{abstract}

Key words: global financial crisis, international stock markets, EGARCH, contagion effect. 


\section{Egitania \\ s c i e $\Omega$ c i a}

\section{RESUMEN:}

En este trabajo se estudia el impacto de la crisis financiera global al nível del contagio entre los mercados bolsistas. Con este objetivo, fueron seleccionados doce de los mayores mercados bolsistas, europeos y no europeos, y fue escogido el periodo comprendido entre el 4/10/1999 y el 30/06/2011. Para identificar la ocurrencia del efecto de contagio, se recurrió al modelo exponencial de heterocedasticidad condicionada (EGARCH) y a testes a los coeficientes de correlación, de modo a entender si los coeficientes registrados en el subperíodo Crise Financeira Global difieren de los registrados en los subperíodos anteriores. Las conclusiones obtenidas revelan que los coeficientes de correlación sufrieron un aumento significativo no último subperíodo, lo que confirma a existencia de efectos de contagio entre los mercados bolsistas estudiados.

Palabras-llave: Crisis financiera global, mercados bolsistas internacionales, EGARCH, efecto de contagio.

*Professor do Instituto Politécnico da Guarda

** Professor da Universidade da Beira Interior 


\section{Eqitania \\ s c i e $\Omega$ c i a}

\section{INTRODUÇÃO}

Na última década vários episódios de crise marcaram os mercados financeiros. Os dois episódios mais marcantes foram o da crise das empresas tecnológicas (dot-com) e o da crise financeira global, desencadeada nos EUA, no setor do crédito subprime, a qual foi considerada por alguns autores como a primeira crise global e a mais severa crise desde a Grande Depressão (Claessens et al., 2010; Bekaert et al., 2011; e Lin e Treichel, 2012). Embora esta crise tivesse a sua origem nos Estados Unidos, acabaria por se transmitir a outros setores económicos, bem como a outras economias, quer desenvolvidas quer emergentes. Também viria a afetar as bolsas mundiais, com muitas praças bolsistas a viverem crashes mais acentuados do que o vivido no país onde emergiu.

Com o objetivo de estudar a existência de um eventual fenómeno de contágio internacional, decorrente da emergência da recente crise financeira global, adotámos uma abordagem diferenciada, que resulta da conjugação das propostas de Lin et al. (1994) e de Forbes e Rigobon (2002), de acordo com a qual o fenómeno de contágio se manifesta no reforço das ligações entre os mercados, designadamente através do risco evidenciado por estes. Adicionalmente, foi escolhida uma amostra diversificada, que inclui mercados desenvolvidos e emergentes, de diversas geografias, de modo a formar uma conclusão mais sólida acerca do efeito da crise financeira global no comportamento dos mercados bolsistas internacionais.

O presente estudo está estruturado do seguinte modo: a parte 2 expõe alguns dos principais trabalhos acerca da temática do contágio; a parte 3 apresenta a informação acerca dos dados e da metodologia escolhida; a parte 4 apresenta os resultados empíricos, enquanto na parte 5 são expostas as principais conclusões.

\section{REVISÃO DA LITERATURA}

O conceito de contágio tem merecido a atenção de diversos autores, não existindo porém consenso acerca do mesmo. Contudo, a maioria dos estudos refere a mudança nos padrões de correlação dos mercados financeiros como um elemento que confirma a ocorrência de contágio.

Calvo e Reinhart (1996) e Park e Song (2000) definem contágio como a propagação de perturbações entre mercados.

Alguns autores, como por exemplo Eichengreen e Rose (1998) e Glick e Rose (1999), apresentam uma definição mais abrangente, incluindo a transmissão de choques entre economias, através dos canais de contágio.

Masson (1999), Van Rijckeghem e Weder (2001) e Rigobon (2003), entre outros autores, apresentam uma definição mais restrita de contágio, considerando unicamente canais de 


\section{Eqitania \\ $s$ c i e $\Omega$ c i a}

transmissão específicos e choques exacerbados. Neste caso, o movimento simultâneo de variáveis económicas, em períodos de turbulência, é um sintoma de contágio.

Lin et al. (1994) associam a definição de contágio à transmissão da volatilidade dos preços dos ativos financeiros, de um dado país em crise, aos mercados financeiros de outros países.

Para King e Wadhwani (1990) e Collins e Biekpe (2003), o contágio financeiro é observado através da intensificação das correlações entre os mercados financeiros, durante períodos de turbulência ou de crise financeira.

De acordo com Forbes e Rigobon (2002), a definição mais consensual atribuída ao termo contágio é a de um aumento significativo no comovimento entre os mercados, após um choque sobre um determinado país ou grupo de países. Neste sentido se, após um choque, dois mercados são altamente correlacionados, isso não é necessariamente contágio. Se anteriormente ao choque, a correlação entre eles já era alta, e se não houve uma alteração significativa em tal correlação, estes mercados poderiam ser considerados interdependentes, ou seja, não teria ocorrido contágio. Estes autores utilizam, então, o termo contágio por alteração (shift-contagion) para diferenciar a sua definição das demais.

\section{DADOS, METODOLOGIA E HIPÓTESES DE INVESTIGAÇÃO}

\subsection{DADOS}

Com o propósito de estudar a eventual existência de contágio entre os mercados bolsistas, foram selecionados índices representativos dos mercados internacionais, concretamente índices europeus, não europeus, desenvolvidos e emergentes, de acordo com a classificação atribuída pela Morgan Stanley Capital International. O conjunto de mercados desenvolvidos incluiu mercados europeus e não europeus. Do contexto europeu, foram escolhidos os mercados da Alemanha (DAX 30), da França (CAC 40), da Inglaterra (FTSE 100), da Espanha (IBEX 35), da Irlanda (ISEQ Overall), da Grécia (ATG) e de Portugal (PSI 20). No conjunto de mercados desenvolvidos não europeus foram considerados os mercados dos EUA (Dow Jones), do Japão (Nikkei 225) e de Hong-Kong (Hang-Seng). Relativamente aos mercados emergentes, foram selecionados o Brasil (Bovespa) e a Índia (Sensex).

Os dados utilizados neste estudo foram obtidos junto da Econostats e cobrem o período compreendido entre 4 de outubro de 1999 e 30 de junho de 2011, que por sua vez foi subdividido em três subperíodos. Para analisar a crise Dot-Com, foi considerado o período de 4/10/1999 a 31/03/2003. Relativamente ao mais recente episódio de crise financeira, designado no âmbito do presente trabalho por Crise Financeira Global, e que foi desencadeado nos EUA, com a crise do crédito subprime, considerou-se que este teve o seu início no dia 1/08/2007, apontado como o momento em que os mercados financeiros 


\section{Egitania}

s c i e $\Omega$ c i a

internacionais foram surpreendidos pela crise subprime, em consequência da acentuada subida dos CDS's (Horta et al., 2008; Toussaint, 2008; e Naoui et al., 2010). Para além dos períodos de crise, foi ainda considerado um terceiro período, que corresponde a uma situação de uma certa estabilidade de mercado, de 1/04/2003 a 31/07/2007, ou seja, ao horizonte temporal compreendido entre os dois episódios de crise financeira.

As séries dos valores de fecho dos índices foram transformadas em séries de rendibilidades logarítmicas, instantâneas ou compostas continuamente, $r_{t}$, através da seguinte expressão:

$r_{t}=1 \quad P_{t}-1 \quad P_{t-1}$

Em que $r_{t}$ é a taxa de rendibilidade, no dia $t$, e $P_{t}$ e $P_{t-1}$ são os valores de fecho das séries, nos momentos $t$ e $t-1$, respetivamente.

\subsection{METODOLOGIA E HIPÓTESES DE INVESTIGAÇÃO}

\subsubsection{MODELOS DE HETEROCEDASTICIDADE CONDICIONADA}

O modelo AutoRegressive Conditional Heterocedasticity (ARCH), proposto por Engle (1982), veio suprir uma falha dos modelos tradicionais, que pressupunham homocedasticidade entre os erros. Este pressuposto mostrava-se desajustado, principalmente em momentos de elevada turbulência ou agitação dos mercados.

Uma evolução do modelo de Engle (1982) foi preconizada por Nelson (1991), ao propor o modelo EGARCH, também denominado por GARCH exponencial. A introdução deste modelo permitiu colmatar a principal limitação dos modelos simétricos, no que respeita à acomodação da ocorrência de choques sobre a volatilidade, razão pela qual optámos por recorrer a este modelo no presente trabalho. Neste modelo, a variância condicionada é descrita por uma função assimétrica dos valores passados de $\varepsilon_{\mathrm{t}}$.

A equação da variância, de acordo com a especificação do modelo EGARCH (p,q), é dada por:

$1 \quad\left(\sigma^{2}\right)=c_{0}+\sum_{i=1}^{q} \alpha_{i} \frac{\left|\varepsilon_{t-i}\right|}{\sigma_{t-i}}+\sum_{k=1}^{r} \gamma_{k} \frac{\varepsilon_{t-k}}{\sigma_{t-k}}+\sum_{j=1}^{p} \beta_{j} l \quad\left(\sigma_{-j}^{2}\right)$ 
Onde:

$c_{0}$ é a constante do modelo, $\alpha_{\mathrm{i}}$ e $\beta_{\mathrm{i}}$ traduzem os efeitos $\mathrm{ARCH}$ e $\mathrm{GARCH}$, respetivamente, e $\gamma_{\mathrm{k}}$ mede o efeito assimétrico; q é a ordem do processo $\mathrm{ARCH}$; p é a ordem do processo $\mathrm{GARCH}$ e $\varepsilon_{\mathrm{t}}$ corresponde ao vetor dos resíduos estimados.

Metodologicamente, optou-se pelo modelo EGARCH (1,1), que define a ordem 1 para os efeitos ARCH e GARCH. Diversos autores, entre os quais Brooks (2002) e Hansen e Lunde (2005), defendem que só raramente os modelos de ordem superior descrevem melhor a volatilidade, razão que justificou a escolha do modelo mais simples.

Para se perceber da correta especificação dos modelos escolhidos, recorremos aos testes de Ljung-Box e ARCH-LM. O teste de Ljung-Box pressupõe que, sob a hipótese nula, $H_{0}: \rho_{1}=\left(\varepsilon_{t}^{2}\right)=\cdots=\rho_{m}=\left(\varepsilon_{t}^{2}\right)=0$, os resíduos quadráticos estandardizados não se encontram correlacionados. $\rho_{i}=\left(\varepsilon_{t}^{2}\right)$ refere-se ao coeficiente de correlação entre $\varepsilon_{t}^{2}$ e $\varepsilon_{t-i}^{2}$, e $\varepsilon_{t}^{2}=u_{t}^{2} / \sigma_{t}^{2}$ diz respeito aos resíduos quadráticos estandardizados. $\bigcirc$ valor da estatística de Ljung-Box é dado por: $Q=n(n+2) \sum_{i=1}^{m} \frac{\hat{\rho}_{i}^{2}\left(\hat{\varepsilon}_{t}^{2}\right)}{n-i} \sim \chi_{(m-k)}^{2}$, em que $k$ traduz o número de parâmetros estimados.

Já o teste $\mathrm{ARCH}$-LM considera que, sob a hipótese nula, $H_{0}: \alpha_{1}=\alpha_{2}=\cdots=\alpha_{q}$, em que $q$ expressa a ordem do processo. A estatística teste é dada por $N R^{2}$, que segue assimptoticamente a distribuição do $\chi^{2}$, com $q$ graus de liberdade, em que $R^{2}$ representa o coeficiente de determinação da regressão e $N$ o número de observações.

\subsubsection{COEFICIENTES DE CORRELAÇÃo E TESTE dO RÁCIO DE VEROSIMILHANÇA}

Para analisar as ligações entre os mercados bolsistas, são consideradas duas medidas estatísticas.

A primeira medida é proporcionada pelo coeficiente de correlação, calculado com base nas estimativas de volatilidade condicionada, produzidas pelo modelo $\mathrm{EGARCH}(1,1)$, e que proporciona uma medida acerca da significância individual de cada par de índices.

O coeficiente de correlação é dado pela habitual medida

$$
r=\frac{\left.\sum\left(x_{i}-\bar{x}\right) y_{i}-\bar{y}\right)}{\left[\sum\left(x_{i}-\bar{x}\right)^{2} \Sigma\left(y_{i}-\bar{y}\right)^{2}\right]^{\frac{1}{2}}}
$$




\section{Eqitania}

$s$ c i e $\Omega$ c i a

Pestana e Gageiro (2000) interpretam a intensidade da correlação, entre duas variáveis, através de um "guia" que atende ao valor numérico desta. A interpretação é feita em cinco níveis: $0 \leq|R|<0,20$ - correlação muito fraca; $0,20 \leq|R|<0,40$ - correlação fraca; $0,40 \leq|R|<0,70$ - correlação moderada; $0,70 \leq|R|<0,90$ - correlação forte; $0,90 \leq|R| \leq 1,00$ correlação muito forte.

Para testar a significância estatística do coeficiente de correlação, recorre-se à estatística $t=\frac{r \cdot \sqrt{n-2}}{\sqrt{1-r^{2}}}$, que segue a distribuição $t$, com $n-2$ graus de liberdade, em que $n$ é o coeficiente de correlação entre duas séries e é o número de observações.

A segunda medida é fornecida pelo teste do rácio de verosimilhança, sugerido por Pindyck e Rotemberg (1990), que permite testar se a matriz de coeficientes de correlação é globalmente diferente da matriz identidade, proporcionando uma indicação da significância conjunta da matriz de correlações. A hipótese nula deste teste pressupõe que não existe correlação entre os vários mercados da amostra. A estatística do teste é dada por $t=-N \cdot 1 \quad \phi R \mid$, que segue uma distribuição Qui-Quadrado, com $0,5 p(p-1)$ graus de liberdade, em que $|R|$ é o determinante da matriz das correlações, $N$ é o número de observações na amostra comum e $p$ é o número de séries analisadas no teste.

\subsubsection{TESTES-T EM DUAS AMOSTRAS E HIPÓTESES DE INVESTIGAÇÃO}

No sentido de perceber da eventual ocorrência de um fenómeno de contágio durante a crise financeira global, recorremos o teste-t em duas amostras, também designado de teste-t de heterocedasticidade, seguindo a proposta de Forbes e Rigobon (2002), que envolve a comparação de correlações relativas a dois períodos temporais, traduzida nas hipóteses de investigação definidas em 4 e 5, que correspondem à hipótese nula de que a correlação no subperíodo Crise Financeira Global é maior ou igual que a correlação nos dois subperíodos anteriores, contra a hipótese alternativa de que a correlação é superior nos dois subperíodos precedentes, e que nortearam a investigação desenvolvida no presente trabalho.

$$
\begin{gathered}
H_{0}=r_{i, j}^{1} \geq r_{i, j}^{0} \\
H_{1}=r_{i, j}^{1}<r_{i, j}^{0}
\end{gathered}
$$

Onde $r_{i, j}^{t}$ é o coeficiente de correlação entre o mercado i e o mercado j, no período t. Nas hipóteses anteriores (4 e 5), ao subperíodo Crise Financeira Global corresponde o valor "1", enquanto aos subperíodos precedentes corresponde o valor "O".

A aplicação do teste de Forbes e Rigobon (2002) considera a transformação de Fisher, aplicada aos coeficientes de correlação, de tal forma que estes apresentam uma distribuição 
aproximadamente normal, em termos assimptóticos, com média $\mu_{t}$ e variância $\sigma_{t}^{2}$, definidas da seguinte forma:

$$
\begin{aligned}
& \mu_{t}=\frac{1}{2} l\left(h \frac{1+r_{i, j}^{t}}{1-r_{i, j}^{t}}\right) \\
& \sigma_{t}^{2}=\frac{1}{n_{t}-3}
\end{aligned}
$$

A estatística do teste é calculada a partir de

$$
U=\frac{\bar{\mu}_{1}-\bar{\mu}_{0}}{\left(\sigma_{0}^{2}+\sigma_{1}^{2}\right)^{\frac{1}{2}}}
$$

Onde $\mu_{t}$ e $\sigma_{t}^{2}$ são a média e a variância amostrais transformadas. A estatística do teste segue uma distribuição normal, com média 0 e variância 1.

\section{RESULTADOS EMPÍRICOS}

Para estimar a volatilidade condicionada, recorreu-se ao modelo EGARCH $(1,1)$, que foi aplicado às rendibilidades logarítmicas dos doze índices, cujos resultados são apresentados na tabela 1. Em geral os parâmetros do modelo evidenciam elevado significância estatística. É, contudo, de destacar o parâmetro relativo ao efeito assimétrico, que é capturado pelo coeficiente $\gamma$. Quando o coeficiente assimétrico tem sinal negativo, os choques positivos produzem volatilidade menos acentuada do que os choques negativos de idêntica dimensão. Das estimativas dos modelos EGARCH $(1,1)$, conclui-se que todos os coeficientes $\gamma$ apresentaram sinal negativo, ou seja, evidenciaram presença de efeito assimétrico. Adicionalmente, nos três subperíodos, estes coeficientes revelaram ser estatisticamente diferentes de zero, ao nível de significância de 1\%. As exceções foram o índice HANG, no subperíodo Tranquilo, que apresentou significância estatística, ao nível de significância de 5\%, e o índice PSI, no subperíodo Tranquilo, cujo coeficiente de assimetria não se revelou estatisticamente diferente de zero. A elevada significância dos coeficientes de assimetria mostra, claramente, a existência de choques assimétricos na volatilidade das rendibilidades 
diárias dos índices, com os choques negativos a produzirem volatilidade mais acentuada do que os choques positivos de idêntica dimensão.

Tabela 1: Resultados da estimação da volatilidade condicionada por modelos $\operatorname{EGARCH}(1,1)$

\begin{tabular}{|c|c|c|c|c|c|c|c|c|c|}
\hline & \multicolumn{3}{|c|}{ ATG } & \multicolumn{3}{|c|}{ BOV } & \multicolumn{3}{|c|}{ CAC } \\
\hline & Dot-Com & Tranq. & C. Global & Dot-Com & Tranq. & C. Global & Dot-Com & Tranq. & C. Global \\
\hline \multirow{2}{*}{$c_{0}$} & $-1,151$ & $-0,455$ & $-0,378$ & $-1,063$ & $-2,020$ & $-0,221$ & $-0,301$ & $-0,332$ & $-0,375$ \\
\hline & $(0,000)$ & $(0,000)$ & $(0,000)$ & $(0,014)$ & $(0,000)$ & $(0,000)$ & $(0,000)$ & $(0,000)$ & $(0,000)$ \\
\hline \multirow{2}{*}{$\alpha_{1}$} & 0,341 & 0,154 & 0,165 & 0,114 & 0,027 & 0,146 & 0,137 & 0,068 & 0,139 \\
\hline & $(0,000)$ & $(0,000)$ & $(0,000)$ & $(0,036)$ & $(0,474)$ & $(0,000)$ & $(0,000)$ & $(0,001)$ & $(0,000)$ \\
\hline \multirow[t]{2}{*}{$\gamma$} & $-0,100$ & $-0,044$ & $-0,079$ & $-0,074$ & $-0,238$ & $-0,090$ & $-0,055$ & $-0,129$ & $-0,194$ \\
\hline & $(0,000)$ & $(0,001)$ & $(0,000)$ & $(0,000)$ & $(0,000)$ & $(0,000)$ & $(0,000)$ & $(0,000)$ & $(0,000)$ \\
\hline \multirow[t]{4}{*}{$\beta$} & 0,894 & 0,963 & 0,969 & 0,875 & 0,758 & 0,987 & 0,977 & 0,970 & 0,969 \\
\hline & $(0,000)$ & $(0,000)$ & $(0,000)$ & $(0,000)$ & $(0,000)$ & $(0,000)$ & $(0,000)$ & $(0,000)$ & $(0,000)$ \\
\hline & \multicolumn{3}{|c|}{ DAX } & \multicolumn{3}{|c|}{ DJ } & \multicolumn{3}{|c|}{ FTSE } \\
\hline & Dot-Com & Tranq. & C. Global & Dot-Com & Tranq. & C. Global & Dot-Com & Tranq. & C. Global \\
\hline \multirow{2}{*}{$c_{0}$} & $-0,372$ & $-0,338$ & $-0,327$ & $-0,239$ & $-0,579$ & $-0,349$ & $-0,306$ & $-0,365$ & $-0,291$ \\
\hline & $(0,000)$ & $(0,000)$ & $(0,000)$ & $(0,002)$ & $(0,000)$ & $(0,000)$ & $(0,000)$ & $(0,000)$ & $(0,000)$ \\
\hline \multirow{2}{*}{$\alpha_{1}$} & 0,187 & 0,103 & 0,142 & 0,056 & 0,075 & 0,142 & 0,139 & 0,072 & 0,114 \\
\hline & $(0,000)$ & $(0,000)$ & $(0,000)$ & $(0,035)$ & $(0,001)$ & $(0,000)$ & $(0,000)$ & $(0,004)$ & $(0,000)$ \\
\hline \multirow[t]{2}{*}{$\gamma$} & $-0,049$ & $-0,111$ & $-0,155$ & $-0,112$ & $-0,107$ & $-0,147$ & $-0,094$ & $-0,125$ & $-0,149$ \\
\hline & $(0,002)$ & $(0,000)$ & $(0,000)$ & $(0,000)$ & $(0,000)$ & $(0,000)$ & $(0,000)$ & $(0,000)$ & $(0,000)$ \\
\hline \multirow[t]{4}{*}{$\beta$} & 0,972 & 0,971 & 0,975 & 0,978 & 0,947 & 0,973 & 0,978 & 0,968 & 0,977 \\
\hline & $(0,000)$ & $(0,000)$ & $(0,000)$ & $(0,000)$ & $(0,000)$ & $(0,000)$ & $(0,000)$ & $(0,000)$ & $(0,000)$ \\
\hline & \multicolumn{3}{|c|}{ HANG-SENG } & \multicolumn{3}{|c|}{ IBEX } & \multicolumn{3}{|c|}{ ISEQ } \\
\hline & Dot-Com & Tranq. & C. Global & Dot-Com & Tranq. & C. Global & Dot-Com & Tranq. & C. Global \\
\hline \multirow{2}{*}{$c_{0}$} & $-0,290$ & $-0,192$ & $-0,259$ & $-0,335$ & $-1,039$ & $-0,333$ & $-0,734$ & $-0,968$ & $-0,349$ \\
\hline & $(0,001)$ & $(0,006)$ & $(0,000)$ & $(0,000)$ & $(0,000)$ & $(0,000)$ & $(0,000)$ & $(0,000)$ & $(0,000)$ \\
\hline \multirow{2}{*}{$\alpha_{1}$} & 0,147 & 0,072 & 0,178 & 0,109 & 0,138 & 0,147 & 0,120 & 0,134 & 0,221 \\
\hline & $(0,000)$ & $(0,000)$ & $(0,000)$ & $(0,003)$ & $(0,000)$ & $(0,000)$ & $(0,000)$ & $(0,000)$ & $(0,000)$ \\
\hline \multirow[t]{2}{*}{$\gamma$} & $-0,060$ & $-0,018$ & $-0,066$ & $-0,085$ & $-0,160$ & $-0,162$ & $-0,124$ & $-0,135$ & $-0,071$ \\
\hline & $(0,000)$ & $(0,040)$ & $(0,000)$ & $(0,000)$ & $(0,000)$ & $(0,000)$ & $(0,000)$ & $(0,000)$ & $(0,000)$ \\
\hline \multirow[t]{4}{*}{$\beta$} & 0,979 & 0,985 & 0,985 & 0,970 & 0,902 & 0,974 & 0,928 & 0,908 & 0,978 \\
\hline & $(0,000)$ & $(0,000)$ & $(0,000)$ & $(0,000)$ & $(0,000)$ & $(0,000)$ & $(0,000)$ & $(0,000)$ & $(0,000)$ \\
\hline & \multicolumn{3}{|c|}{ NIKKEI } & \multicolumn{3}{|c|}{ PSI } & \multicolumn{3}{|c|}{ SENSEX } \\
\hline & Dot-Com & Tranq. & C. Global & Dot-Com & Tranq. & C. Global & Dot-Com & Tranq. & C. Global \\
\hline$c_{0}$ & $-0,560$ & $-0,502$ & $-0,426$ & $-1,293$ & $-0,489$ & $-0,558$ & $-0,981$ & $-1,229$ & $-0,308$ \\
\hline 0 & 0,002 & $(0,000)$ & $(0,000)$ & $(0,000)$ & $(0,000)$ & $(0,000)$ & $(0,000)$ & $(0,000)$ & $(0,000)$ \\
\hline$\alpha_{1}$ & 0,146 & 0,171 & 0,196 & 0,268 & 0,118 & 0,225 & 0,284 & 0,274 & 0,220 \\
\hline & $(0,000)$ & $(0,000)$ & $(0,000)$ & $(0,000)$ & $(0,000)$ & $(0,000)$ & $(0,000)$ & $(0,000)$ & $(0,000)$ \\
\hline$\gamma$ & $-0,055$ & $-0,078$ & $-0,126$ & $-0,108$ & $-0,005$ & $-0,134$ & $-0,125$ & $-0,172$ & $-0,074$ \\
\hline & $(0,009)$ & $(0,000)$ & $(0,000)$ & $(0,000)$ & 0,735 & $(0,000)$ & $(0,000)$ & $(0,000)$ & $(0,000)$ \\
\hline$\beta$ & 0,947 & 0,959 & 0,968 & 0,880 & 0,961 & 0,957 & 0,908 & 0,882 & 0,983 \\
\hline & $(0,000)$ & $(0,000)$ & $(0,000)$ & $(0,000)$ & $(0,000)$ & $(0,000)$ & $(0,000)$ & $(0,000)$ & $(0,000)$ \\
\hline
\end{tabular}

Notas: Esta tabela apresenta os resultados de estimação do modelo EGARCH $(1,1)$, relativos aos três subperíodos amostrais (Dot-Com: de 4/10/1999 a 31/03/2003; Tranquilo: de 1/04/2003 a 31/07/2007; Crise Financeira Global: de 1/08/2007 a 30/06/2011) e aos doze mercados estudados, designadamente Grécia (ATG), Brasil (BOV), França (CAC), Alemanha (DAX), EUA (DJ), Inglaterra (FTSE), Hong-Kong (HANG-SENG), Espanha (IBEX), Irlanda (ISEQ), Japão (NIIKKEI), Portugal (PSI) e Índia (SENSEX). Os valores entre parêntesis indicam o p-value de cada um dos parâmetros do modelo estimado. 
Para averiguar da correta especificação dos modelos, foi analisado o comportamento dos resíduos, de modo a perceber se estes apresentaram um comportamento idêntico ao de um processo ruído branco. Com esse intuito, recorreu-se aos testes de Ljung-Box e ARCH-LM, cujas estatísticas são mostradas na tabela 2. Os resultados dos testes de Ljung-Box, aos resíduos dos modelos EGARCH $(1,1)$, permitiram concluir que, relativamente aos índices DAX (subperíodo Dot-Com), BOV (subperíodo Tranquilo) e HANG (subperíodo Crise Financeira Global), não se aceita a hipótese nula, pois os valores dos p-value são bastante superiores ao nível de significância de 5\%, ou seja, a correlação do quadrado dos resíduos não é ruído branco. Quanto aos restantes índices, há uma forte evidência de não rejeição da hipótese nula, concluindo-se que os resíduos estandardizados não estão correlacionados. Estas conclusões foram confirmadas com a aplicação do teste LM (tabela 2). O teste aos coeficientes em grupo mostra que a probabilidade (F-Statistic) não é significativa nos casos referidos anteriormente, rejeitando-se a hipótese nula. Nesses casos, o modelo EGARCH $(1,1)$ mostrou que os resíduos das séries não foram suficientemente branqueados. Nos restantes casos, todos os valores da estatística do teste superaram, claramente, o nível de significância de $5 \%$.

Tabela 2: Resultados dos testes de Ljung-Box e LM aos resíduos dos modelos $\operatorname{EGARCH}(1,1)$

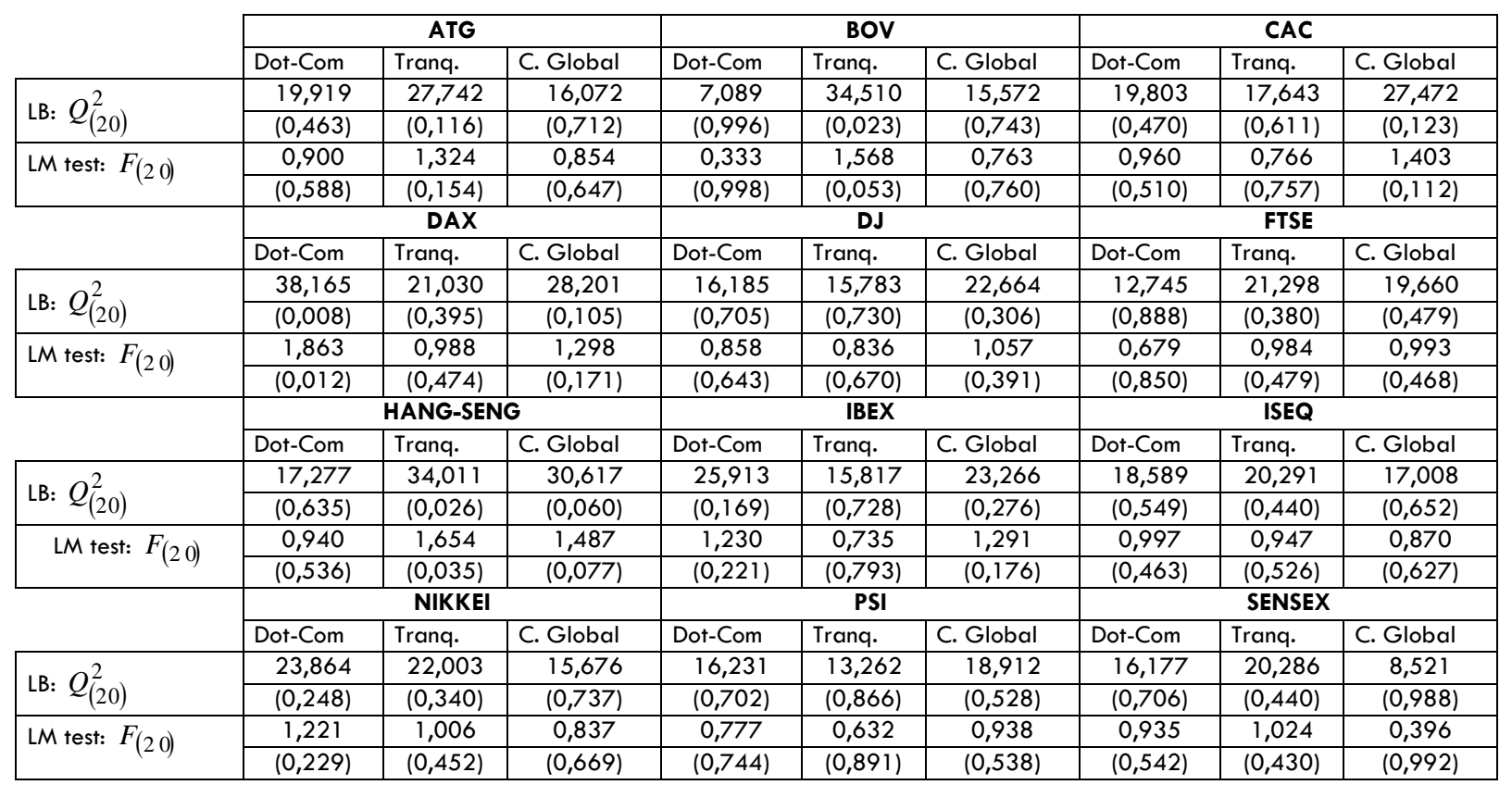

Notas: Esta tabela apresenta os resultados dos testes de Ljung-Box e ARCH-LM, aplicados aos resíduos dos modelos EGARCH (1,1), dos índices da Grécia (ATG), Brasil (BOV), França (CAC), Alemanha (DAX), EUA (DJ), Inglaterra (FTSE), Hong-Kong (HANG-SENG), Espanha (IBEX), Irlanda (ISEQ), Japão (NIKKEI), Portugal (PSI) e Índia (SENSEX), nos três subperíodos (Dot-Com: de 4/10/1999 a 31/03/2003; Tranquilo: de 1/04/2003 a 31/07/2007; Crise Financeira Global: de 1/08/2007 a 30/06/2011), e considerando em ambos os casos o lag 20. Os valores entre parêntesis correspondem aos valores de probabilidade de cada um dos testes. 
Para estudar a existência de comovimentos de curto prazo entre a volatilidade condicionada diária dos diversos mercados, em cada um dos subperíodos amostrais, calcularam-se os coeficientes de correlação das estimativas de volatilidade condicionada, obtidas através dos modelos EGARCH $(1,1)$, bem como os rácios de verosimilhança das matrizes de coeficientes de correlação, de acordo com a sugestão de Pindyck e Rotemberg (1990), e averiguou-se da significância estatística de ambas as medidas estatísticas. Se a primeira medida fornece uma indicação acerca da significância individual de cada par de índices, a segunda proporciona uma indicação da significância conjunta da matriz de correlações.

Nas tabelas 3, 4 e 5 são mostradas as correlações entre as volatilidades diárias condicionadas dos índices dos vários mercados estudados, bem como os rácios de verosimilhança, considerando as estimativas produzidas pelo modelo $\mathrm{EGARCH}(1,1)$, relativas a cada um dos três subperíodos amostrais.

A análise da matriz de correlações do subperíodo Dot-Com (tabela 3) revela que a esmagadora maioria dos coeficientes de correlação são não nulos e estatisticamente significativos, ao nível de significância de 5\%. Contudo, alguns coeficientes não se mostraram estatisticamente significativos, em especial nos pares que envolveram os índices ATG e SENSEX. As correlações entre as volatilidades dos mercados revelaram-se, em geral, positivas. Apenas os pares ATG-CAC, ATG-DAX, CAC-SENSEX e DAX-SENSEX apresentaram correlação negativa, embora só a correlação do segundo par tivesse significado estatístico, ao nível de significância de 5\%, de acordo com os valores dos p-values.

O sinal positivo dos coeficientes de correlação sugere a existência de uma tendência comum que conduz a volatilidade dos mercados na mesma direção. Porém, diversos pares de índices apresentaram correlações muito fracas, inferiores a 0.2, em especial envolvendo o índice ATG. Em sentido oposto, oito pares registaram correlações fortes (sete pares europeus) e dois registaram correlações muito fortes, designadamente os pares CAC-DAX e CAC-FTSE, ambos com uma correlação de 0,93.

No subperíodo Tranquilo, todos os pares de índices reportaram correlações positivas e estatisticamente significativas, ao nível de significância de 1\%, tendo em conta as respetivas probabilidades (tabela 4). Neste período, apenas os pares que envolveram o índice BOV com os índices DAX, HANG, NIKKEI e PSI apresentaram uma correlação muito fraca. Dezassete pares registaram uma correlação fraca. A maioria dos pares de índices registou uma correlação moderada, sete registaram uma correlação forte, enquanto os pares CAC-DAX e CAC-FTSE tiveram uma correlação muito forte. 
Tabela 3: Correlações contemporâneas entre as volatilidades condicionais diárias, no subperíodo Dot-Com

\begin{tabular}{|c|c|c|c|c|c|c|c|c|c|c|c|c|}
\hline & ATG & $\mathrm{BOV}$ & CAC & DAX & DJ & FTSE & HANG & IBEX & ISEQ & NIKKEI & PSI & SENSEX \\
\hline $\begin{array}{l}\text { ATG } \\
\text { BOV }\end{array}$ & $\begin{array}{c}1 \\
0,256 \\
(0,000)\end{array}$ & 1 & & & & & & & & & & \\
\hline CAC & $\begin{array}{l}-0,060 \\
(0,082)\end{array}$ & $\begin{array}{c}0,400 \\
(0,000)\end{array}$ & 1 & & & & & & & & & \\
\hline DAX & $\begin{array}{l}-0,101 \\
(0,003)\end{array}$ & $\begin{array}{c}0,385 \\
(0,000)\end{array}$ & $\begin{array}{c}0,933 \\
(0,000)\end{array}$ & 1 & & & & & & & & \\
\hline DJ & $\begin{array}{c}0,106 \\
(0,002)\end{array}$ & $\begin{array}{c}0,514 \\
(0,000)\end{array}$ & $\begin{array}{c}0,794 \\
(0,000)\end{array}$ & $\begin{array}{c}0,764 \\
(0,000)\end{array}$ & 1 & & & & & & & \\
\hline FTSE & $\begin{array}{c}0,012 \\
(0,723)\end{array}$ & $\begin{array}{c}0,427 \\
(0,000)\end{array}$ & $\begin{array}{c}0,926 \\
(0,000)\end{array}$ & $\begin{array}{c}0,865 \\
(0,000)\end{array}$ & $\begin{array}{c}0,832 \\
(0,000)\end{array}$ & 1 & & & & & & \\
\hline HANG & $\begin{array}{c}0,401 \\
(0,000)\end{array}$ & $\begin{array}{r}0,506 \\
(0,000)\end{array}$ & $\begin{array}{c}0,1112 \\
(0,001)\end{array}$ & $\begin{array}{c}0,078 \\
(0,023)\end{array}$ & $\begin{array}{c}0,303 \\
(0,000)\end{array}$ & $\begin{array}{c}0,198 \\
(0,000)\end{array}$ & 1 & & & & & \\
\hline IBEX & $\begin{array}{c}0,006 \\
(0,872)\end{array}$ & $\begin{array}{c}0,536 \\
(0,000)\end{array}$ & $\begin{array}{c}0,848 \\
(0,000)\end{array}$ & $\begin{array}{c}0,777 \\
(0,000)\end{array}$ & $\begin{array}{c}0,769 \\
(0,000)\end{array}$ & $\begin{array}{c}0,820 \\
(0,000)\end{array}$ & $\begin{array}{c}0,322 \\
(0,000)\end{array}$ & 1 & & & & \\
\hline ISEQ & $\begin{array}{c}0,157 \\
(0,000)\end{array}$ & $\begin{array}{c}0,424 \\
(0,000)\end{array}$ & $\begin{array}{c}0,572 \\
(0,000)\end{array}$ & $\begin{array}{c}0,551 \\
(0,000)\end{array}$ & $\begin{array}{c}0,685 \\
(0,000)\end{array}$ & $\begin{array}{c}0,602 \\
(0,000)\end{array}$ & $\begin{array}{c}0,318 \\
(0,000)\end{array}$ & $\begin{array}{c}0,633 \\
(0,000)\end{array}$ & 1 & & & \\
\hline NIKKEI & $\begin{array}{c}0,1112 \\
(0,001)\end{array}$ & $\begin{array}{c}0,514 \\
(0,000)\end{array}$ & $\begin{array}{c}0,467 \\
(0,000)\end{array}$ & $\begin{array}{c}0,435 \\
(0,000)\end{array}$ & $\begin{array}{c}0,488 \\
(0,000)\end{array}$ & $\begin{array}{c}0,460 \\
(0,000)\end{array}$ & $\begin{array}{c}0,442 \\
(0,000)\end{array}$ & $\begin{array}{c}0,590 \\
(0,000)\end{array}$ & $\begin{array}{c}0,550 \\
(0,000)\end{array}$ & 1 & & \\
\hline PSI & $\begin{array}{c}0,334 \\
(0,000)\end{array}$ & $\begin{array}{c}0,658 \\
(0,000)\end{array}$ & $\begin{array}{c}0,359 \\
(0,000)\end{array}$ & $\begin{array}{c}0,337 \\
(0,000)\end{array}$ & $\begin{array}{c}0,427 \\
(0,000)\end{array}$ & $\begin{array}{c}0,364 \\
(0,000)\end{array}$ & $\begin{array}{c}0,506 \\
(0,000)\end{array}$ & $\begin{array}{c}0,442 \\
(0,000)\end{array}$ & $\begin{array}{c}0,419 \\
(0,000)\end{array}$ & $\begin{array}{c}0,323 \\
(0,000)\end{array}$ & 1 & \\
\hline SENSEX & $\begin{array}{c}0,408 \\
(0,000) \\
\end{array}$ & $\begin{array}{r}0,446 \\
(0,000) \\
\end{array}$ & $\begin{array}{r}-0,033 \\
(0,337) \\
\end{array}$ & $\begin{array}{r}-0,044 \\
(0,200) \\
\end{array}$ & $\begin{array}{r}0,243 \\
(0,000) \\
\end{array}$ & $\begin{array}{r}0,055 \\
(0,1112) \\
\end{array}$ & $\begin{array}{c}0,656 \\
(0,000) \\
\end{array}$ & $\begin{array}{r}0,086 \\
(0,012) \\
\end{array}$ & $\begin{array}{r}0,210 \\
(0,000) \\
\end{array}$ & $\begin{array}{c}0,277 \\
(0,000) \\
\end{array}$ & $\begin{array}{c}0,451 \\
(0,000) \\
\end{array}$ & 1 \\
\hline & $\begin{array}{l}\text { Notas: } \\
\text { Grécia } \\
\text { Espanha } \\
(1,1) \text {, pa } \\
\text { parêntes }\end{array}$ & Esta tabela & apresenta & os coeficie & entes de c & relação e & $\begin{array}{l}\text { entre a vola } \\
\text { EUA (DJ), } \\
\text { e Índia (SE } \\
\text { bro de } 199 \\
\text { nça da matr }\end{array}$ & atilidade co & $\begin{array}{l}\text { nndicionad } \\
\text { (FTSE), } \\
\text { stimada at } \\
\text { e março } \\
\text { lações ten }\end{array}$ & $\begin{array}{l}\text { a relativa ao } \\
\text { Hong-Kong } \\
\text { ravés do ma } \\
\text { de } 2003 \text {. O } \\
\text { o valor de }\end{array}$ & $\begin{array}{l}\text { S mercados } \\
\text { (HANG-SEI } \\
\text { iodelo EGAF } \\
\text { ss valores } \\
9051 .\end{array}$ & $\begin{array}{l}\text { s da } \\
\text { NGG), } \\
\text { RCH } \\
\text { entre }\end{array}$ \\
\hline
\end{tabular}

Tabela 4: Correlações contemporâneas entre as volatilidades condicionais diárias, no subperíodo Tranquilo

\begin{tabular}{|c|c|c|c|c|c|c|c|c|c|c|c|c|}
\hline & ATG & $\mathrm{BOV}$ & CAC & DAX & DJ & FTSE & HANG & IBEX & ISEQ & NIKKEI & PSI & SENSEX \\
\hline ATG & 1 & & & & & & & & & & & \\
\hline \multirow[t]{2}{*}{ BOV } & 0,277 & 1 & & & & & & & & & & \\
\hline & $(0,000)$ & & & & & & & & & & & \\
\hline \multirow[t]{2}{*}{ CAC } & 0,622 & 0,292 & 1 & & & & & & & & & \\
\hline & $(0,000)$ & $(0,000)$ & & & & & & & & & & \\
\hline \multirow[t]{2}{*}{ DAX } & 0,534 & 0,194 & 0,935 & 1 & & & & & & & & \\
\hline & $(0,000)$ & $(0,000)$ & $(0,000)$ & & & & & & & & & \\
\hline \multirow[t]{2}{*}{ DJ } & 0,438 & 0,439 & 0,770 & 0,750 & 1 & & & & & & & \\
\hline & $(0,000)$ & $(0,000)$ & $(0,000)$ & $(0,000)$ & & & & & & & & \\
\hline \multirow[t]{2}{*}{ FTSE } & 0,679 & 0,329 & 0,939 & 0,851 & 0,703 & 1 & & & & & & \\
\hline & $(0,000)$ & $(0,000)$ & $(0,000)$ & $(0,000)$ & $(0,000)$ & & & & & & & \\
\hline \multirow[t]{2}{*}{ HANG } & 0,496 & 0,144 & 0,593 & 0,544 & 0,337 & 0,598 & 1 & & & & & \\
\hline & $(0,000)$ & $(0,000)$ & $(0,000)$ & $(0,000)$ & $(0,000)$ & $(0,000)$ & & & & & & \\
\hline \multirow[t]{2}{*}{ IBEX } & 0,571 & 0,416 & 0,770 & 0,692 & 0,689 & 0,766 & 0,472 & 1 & & & & \\
\hline & $(0,000)$ & $(0,000)$ & $(0,000)$ & $(0,000)$ & $(0,000)$ & $(0,000)$ & $(0,000)$ & & & & & \\
\hline ISEQ & 0,511 & 0,390 & 0,461 & 0,314 & 0,431 & 0,523 & 0,366 & 0,601 & 1 & & & \\
\hline
\end{tabular}




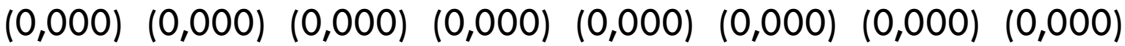

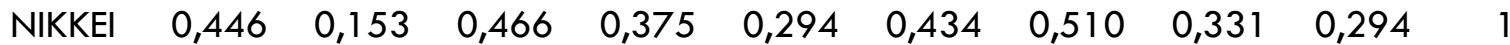

$(0,000)(0,000)(0,000)(0,000)(0,000)(0,000)(0,000)(0,000) \quad(0,000)$

PSI $\quad \begin{array}{lllllllllll}0,451 & 0,136 & 0,655 & 0,711 & 0,578 & 0,638 & 0,564 & 0,552 & 0,262 & 0,256 & 1\end{array}$

$\begin{array}{llllllllll}(0,000) & (0,000) & (0,000) & (0,000) & (0,000) & (0,000) & (0,000) & (0,000) & (0,000) & (0,000)\end{array}$

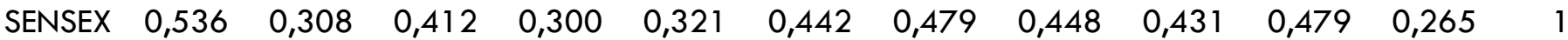
$\begin{array}{lllllllllll}(0,000 & (0,000 & (0,000) & (0,000) & (0,000) & (0,000) & (0,000) & (0,000) & (0,000) & (0,000) & (0,000)\end{array}$

Notas: Esta tabela apresenta os coeficientes de correlação entre a volatilidade condicionada relativa aos mercados da Grécia (ATG), Brasil (BOV), França (CAC), Alemanha (DAX), EUA (DJ), Inglaterra (FTSE), Hong-Kong (HANG-SENG), Espanha (IBEX), Irlanda (ISEQ), Japão (NIKKEI), Portugal (PSI) e Índia (SENSEX), estimada através do modelo EGARCH $(1,1)$, para o subperíodo Tranquilo, de 1 de abril de 2003 a 31 de julho de 2007. Os valores entre parêntesis dizem respeito aos p-values, sendo todos aproximadamente iguais a zero, pelo que todos os coeficientes de correlação são estatisticamente significativos ao nível de 1\%. O rácio de verosimilhança da matriz de correlações tem o valor de 11221.

Tabela 5: Correlações contemporâneas entre as volatilidades condicionais diárias, no subperíodo Crise Financeira Global

\begin{tabular}{|c|c|c|c|c|c|c|c|c|c|c|c|c|}
\hline & ATG & $\mathrm{BOV}$ & CAC & DAX & DJ & FTSE & HANG & IBEX & ISEQ & NIKKEI & PSI & SENSEX \\
\hline ATG & 1 & & & & & & & & & & & \\
\hline BOV & 0,710 & 1 & & & & & & & & & & \\
\hline & $(0,000)$ & & & & & & & & & & & \\
\hline
\end{tabular}

CAC $\quad 0,744 \quad 0,864 \quad 1$

$(0,000)(0,000)$

DAX $\quad 0,722 \quad 0,871 \quad 0,953 \quad 1$

$(0,000)(0,000)(0,000)$

DJ $\quad \begin{array}{lllll}0,667 & 0,921 & 0,915 & 0,934 & 1\end{array}$

$(0,000)(0,000)(0,000) \quad(0,000)$

FTSE $\quad 0,706 \quad 0,920 \quad 0,963 \quad 0,950 \quad 0,940 \quad 1$

$(0,000)(0,000)(0,000)(0,000) \quad(0,000)$

$\begin{array}{llllllll}\text { HANG } & 0,658 & 0,880 & 0,735 & 0,795 & 0,791 & 0,798 & 1\end{array}$

$\begin{array}{llllll}(0,000) & (0,000) & (0,000) & (0,000) & (0,000) & (0,000)\end{array}$

$\begin{array}{lllllllll}\text { IBEX } & 0,840 & 0,811 & 0,914 & 0,858 & 0,819 & 0,865 & 0,726 & 1\end{array}$

$\begin{array}{lllllll}(0,000) & (0,000) & (0,000) & (0,000) & (0,000) & (0,000) & (0,000)\end{array}$

$\begin{array}{llllllllll}\text { ISEQ } & 0,556 & 0,816 & 0,800 & 0,726 & 0,784 & 0,812 & 0,596 & 0,709 & 1\end{array}$

$\begin{array}{llllllll}(0,000) & (0,000) & (0,000) & (0,000) & (0,000) & (0,000) & (0,000) & (0,000)\end{array}$

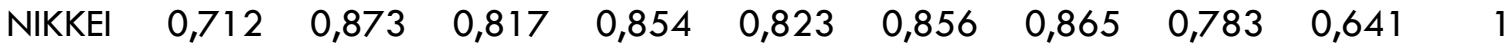

$\begin{array}{llllllllll}(0,000) & (0,000) & (0,000) & (0,000) & (0,000) & (0,000) & (0,000) & (0,000) & (0,000)\end{array}$

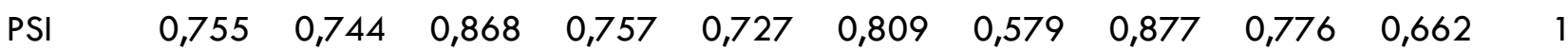

$\begin{array}{llllllllll}(0,000) & (0,000) & (0,000) & (0,000) & (0,000) & (0,000) & (0,000) & (0,000) & (0,000) & (0,000)\end{array}$

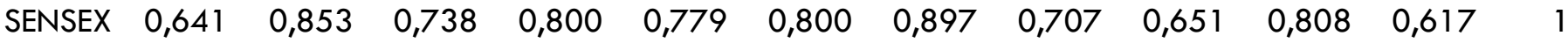

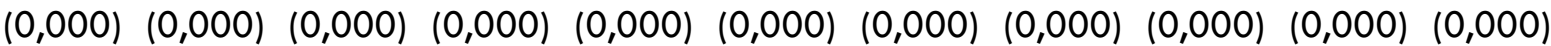

Notas: Esta tabela apresenta os coeficientes de correlação entre a volatilidade condicionada relativa aos mercados da Grécia (ATG), Brasil (BOV), França (CAC), Alemanha (DAX), EUA (DJ), Inglaterra (FTSE), Hong-Kong (HANG-SENG), Espanha (IBEX), Irlanda (ISEQ), Japão (NIKKEI), Portugal (PSI) e India (SENSEX), estimada através do modelo EGARCH $(1,1)$, para o subperíodo da Crise Financeira Global, de 1 de agosto de 2007 a 30 de junho de 2011. Os valores entre parêntesis dizem respeito aos p-values, sendo todos aproximadamente iguais a zero, pelo que todos os coeficientes de 


\section{Equitania}

s c i e $\Omega$ c i a

correlação são estatisticamente significativos ao nível de 1\%. O rácio de verosimilhança da matriz de correlações tem o valor de 21360.

No subperíodo Crise Financeira Global, à semelhança do que aconteceu no subperíodo Tranquilo, todos os pares de índices apresentaram correlações positivas e significativas, ao nível de significância de 1\%, como se pode concluir da análise da tabela 5. Do conjunto de pares de correlações, não foram registadas correlações muito fracas e fracas, dez tiveram uma correlação moderada, quarenta e sete tiveram uma correlação forte, e nove registaram uma correlação muito forte, o que confirma a superioridade das correlações relativamente ao ocorrido nos subperíodos anteriores. Da comparação dos valores das correlações contemporâneas, relativas ao subperíodo Crise Financeira Global e aos dois subperíodos anteriores, conclui-se pela superioridade dos primeiros, em todos os pares de relações lineares.

A intensificação das correlações entre os mercados financeiros, durante a crise financeira global face aos dois subperíodos precedentes, deve ser entendida como um primeiro indicador de eventual ocorrência de contágio financeiro, em consonância com as conclusões formuladas por King e Wadhwani (1990) e Collins e Biekpe (2003), com base no estudo de outros mercados bolsistas e de outros períodos temporais particularmente turbulentos.

Partindo das matrizes de coeficientes de correlação, foram calculados os rácios de verosimilhança. Estes rácios revelaram-se fortemente significativos, ao nível de significância de 1\%, superando o valor crítico de 95,63. Por outro lado, mostraram uma tendência crescente, registando os valores de 9051, 11221 e 21360, nos subperíodos, Dot-com, Tranquilo e Crise Financeira Global, respetivamente. Este facto pode ser entendido como um sinal de que, em geral, as correlações entre os mercados tenderam a aumentar durante o período amostral estudado, pelo que se incrementaram os níveis de associação linear entre os mercados, descrevendo percursos mais semelhantes em termos de volatilidade. $\bigcirc$ aumento no valor desse rácio foi expressivo quer no subperíodo Tranquilo quer no subperíodo Crise Financeira Global. O elevado valor do rácio de verosimilhança, principalmente no último subperíodo, bem como a sua significância estatística, permite uma primeira indicação de que a turbulência vivida pelos mercados estudados possa ter ocorrido em simultâneo.

Com o objetivo de perceber se o aumento generalizado das correlações teve significância estatística, foi aplicado o teste em duas amostras, de acordo com a proposta de Forbes e Rigobon (2002), através do qual é comparado o subperíodo Crise Financeira Global com os dois precedentes. Os resultados deste teste são apresentados na tabela 6. 
Tabela 6: Teste à igualdade das correlações das volatilidades condicionadas diárias (EGARCH)

\begin{tabular}{|c|c|c|c|c|c|c|c|c|c|c|c|}
\hline & \multicolumn{11}{|c|}{ Crise Financeira Global versus Crise Dot-Com } \\
\hline & BOV & CAC & DAX & DJ & FTSE & HANG & IBEX & ISEQ & NIKKEI & PSI & SENSEX \\
\hline $\begin{array}{l}\text { ATG } \\
\text { BOV } \\
\text { CAC } \\
\text { DAX } \\
\text { DJ } \\
\text { FTSE } \\
\text { HANG } \\
\text { IBEX } \\
\text { ISEQ } \\
\text { NIKKEI } \\
\text { PSI } \\
\end{array}$ & 13.28 & $\begin{array}{l}21,64 \\
18,85\end{array}$ & $\begin{array}{c}21,55 \\
19,76 \\
3,88\end{array}$ & $\begin{array}{l}14,86 \\
21,80 \\
10,15 \\
14,50\end{array}$ & $\begin{array}{c}18,42 \\
24,07 \\
7,40 \\
10,99 \\
11,55\end{array}$ & $\begin{array}{l}7,75 \\
17,41 \\
17,57 \\
21,36 \\
16,17 \\
18,95\end{array}$ & $\begin{array}{c}25,82 \\
11,31 \\
6,40 \\
5,26 \\
2,85 \\
3,33 \\
12,45\end{array}$ & $\begin{array}{c}9,96 \\
14,72 \\
9,51 \\
6,38 \\
4,59 \\
9,30 \\
7,61 \\
2,96\end{array}$ & $\begin{array}{c}16,55 \\
16,54 \\
13,60 \\
17,13 \\
13,47 \\
16,56 \\
17,83 \\
7,96 \\
3,00\end{array}$ & $\begin{array}{c}13,55 \\
3,61 \\
20,21 \\
13,58 \\
9,89 \\
15,78 \\
2,20 \\
18,82 \\
12,52 \\
9,80\end{array}$ & $\begin{array}{c}6,94 \\
16,69 \\
20,81 \\
24,26 \\
16,88 \\
22,17 \\
14,30 \\
16,88 \\
11,95 \\
17,76 \\
4,98 \\
\end{array}$ \\
\hline & \multicolumn{11}{|c|}{ Crise Financeira Global versus subperíodo Tranquilo } \\
\hline & BOV & CAC & DAX & DJ & FTSE & HANG & IBEX & ISEQ & NIKKEI & PSI & SENSEX \\
\hline $\begin{array}{l}\text { ATG } \\
\text { BOV } \\
\text { CAC } \\
\text { DAX } \\
\text { DJ } \\
\text { FTSE } \\
\text { HANG } \\
\text { IBEX } \\
\text { ISEQ } \\
\text { NIKKEI } \\
\text { PSI } \\
\end{array}$ & 12.71 & $\begin{array}{c}4,86 \\
21,33\end{array}$ & $\begin{array}{c}6.68 \\
24,08 \\
3,51\end{array}$ & $\begin{array}{l}7,09 \\
23,73 \\
11,37 \\
15,13\end{array}$ & $\begin{array}{c}1,11 \\
26,36 \\
5,35 \\
12,04 \\
18,26\end{array}$ & $\begin{array}{c}5,20 \\
26,02 \\
5,44 \\
10,01 \\
15,27 \\
8,48\end{array}$ & $\begin{array}{r}12,08 \\
14,52 \\
11,17 \\
9,14 \\
6,49 \\
6,37 \\
8,58\end{array}$ & $\begin{array}{c}1,34 \\
15,48 \\
12,65 \\
12,56 \\
12,55 \\
11,68 \\
6,42 \\
4,02\end{array}$ & $\begin{array}{c}8,68 \\
25,18 \\
13,55 \\
18,55 \\
18,24 \\
17,16 \\
15,87 \\
14,94 \\
9,63\end{array}$ & $\begin{array}{c}10,54 \\
17,35 \\
11,45 \\
2,13 \\
5,53 \\
7,80 \\
0,48 \\
15,63 \\
16,20 \\
11,29\end{array}$ & $\begin{array}{c}3,40 \\
19,99 \\
10,75 \\
16,65 \\
15,00 \\
13,15 \\
19,78 \\
8,40 \\
6,66 \\
12,64 \\
9,48 \\
\end{array}$ \\
\hline
\end{tabular}

No subperíodo Crise Financeira Global, face ao subperíodo Dot-Com, os níveis de dependência linear entre os diversos mercados alteraram-se de forma substancial, com todos os pares de correlações a registarem um aumento. Para além disso, em todos os casos, os aumentos revelaram-se estatisticamente significados, ao nível de significância de 5\%, superando o valor crítico de 1,96. Em relação ao subperíodo Tranquilo, os coeficientes de correlação observaram, no subperíodo Crise Financeira Global, um aumento de todos os pares de correlações. Dos aumentos registados, apenas os pares ATG-FTSE, ATG-ISEQ e HANG-PSI não apresentaram um aumento estatisticamente significativo, ao nível de significância de 5\%.

Os resultados obtidos confirmam o reforço das ligações entre os mercados e da ocorrência de um fenómeno de contágio, através da volatilidade condicionada, em resultado da emergência da crise financeira global. A associação entre o aumento de volatilidade dos ativos financeiros e a turbulência financeira é um dos factos estilizados inerentes aos mercados financeiros internacionais. Este facto possibilita que, empiricamente, as crises possam ser 


\section{Egitania}

$s$ c i e $\Omega$ c i a

identificadas com os períodos em que ocorrem volatilidades extremas. Os episódios de contágio estão associados à propagação de volatilidade entre mercados internacionais. Sendo a volatilidade dos preços dos ativos financeiros uma boa aproximação à incerteza do mercado, o fenómeno de contágio pode ser entendido como a expressão da propagação da incerteza entre os mercados financeiros internacionais.

Como tivemos oportunidade de concluir anteriormente, a Crise Financeira contribuiu para um significativo aumento da volatilidade e da turbulência dos mercados financeiros, mas também para um aprofundamento da relação linear entre os mercados. De acordo com a proposta de Forbes e Rigobon (2000), estes factos podem ser entendidos como um fenómeno de contágio por alteração, com implicações ao nível da propagação de risco entre os mercados financeiros. Adicionalmente, foi possível concluir que a Crise Financeira terá atingido proporções globais, em consonância com as conclusões obtidas noutros trabalhos, designadamente os de Claessens et al. (2010), Bekaert et al. (2011) e Lin e Treichel (2012), tendo em consideração o forte efeito de contágio por alteração registado durante o último subperíodo, independentemente do nível de desenvolvimento ou da localização dos mercados bolsistas estudados, dando expressão à grande proximidade entre os mercados bolsistas e à facilidade com que os fenómenos de crise podem transmitir-se internacionalmente.

\section{CONCLUSÃO}

Neste trabalho foi estudada a atual crise financeira, a qual segundo diversos autores é a mais severa crise financeira depois da Grande Depressão e a primeira crise financeira global que o mundo conheceu. De modo a perceber o impacto da crise financeira ao nível do contágio entre os mercados bolsistas, foram analisados doze mercados e foi estudado o período compreendido entre 4/10/1999 e 30/06/2011. Para estudar a ligação entre os mercados, foram calculados coeficientes de correlação, aplicados a estimativas de volatilidade condicionada, e foi considerada a proposta de Forbes e Rigobon (2002), de modo a se identificar um eventual fenómeno de contágio entre os mercados bolsistas.

As conclusões obtidas confirmam que no subperíodo Crise Financeira Global a correlação entre os mercados sofreu um acréscimo significativo, em comparação com os dois subperíodos precedentes. Em relação ao primeiro subperíodo, todos os coeficientes de correlação sofreram um aumento estatisticamente significativo, ao nível de significância de 5\%. Face ao segundo subperíodo, apenas os pares ATG-FTSE, ATG-ISEQ e HANG-PSI não apresentaram um aumento estatisticamente significativo, para o referido nível de significância.

Os resultados permitem concluir que a crise financeira deu origem a um processo de contágio com expressão global, que terá envolvido os mercados bolsistas em geral, sejam eles mercados europeus ou não europeus, ou mesmo mercados desenvolvidos ou emergentes, realçando aquela que parece ser uma caraterística atual dos mercados bolsistas, traduzida na 


\section{Eqitania \\ s c i e $\Omega$ c i a}

sua grande proximidade e interdependência, o que coloca novos e exigentes desafios aos investidores, perante a necessidade de diversificação risco associado aos seus investimentos.

\section{REFERÊNCIAS BIBLIOGRAFICAS}

Bekaert, G., Ehrmann, M., Fratzscher, M. e Mehl, A., (2011), "Global Crises and Equity Market Contagion. National Bureau of EECONOMIC REREARCH", $\quad$ WORKING PaPeR 17121.

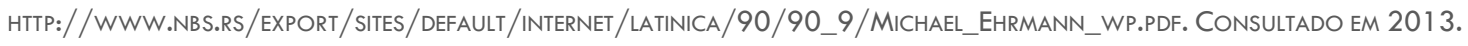

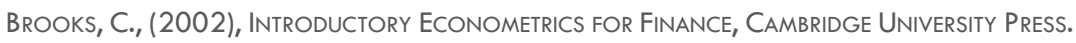

Calvo, S. e Reinhart, C., (1996), "Capital flows to Latin America: is there evidence of contagions effects?", Policy Research WORKING, PAPER 1619, THE WORLD BANK.

Claessens. S., Dell'Ariccia, G., Igan, D. e LaeVen, L., (2010), “Lessons and Policy Implications from the Global Financial Crisis", IMF WORKING PAPER, NO. 10/44.

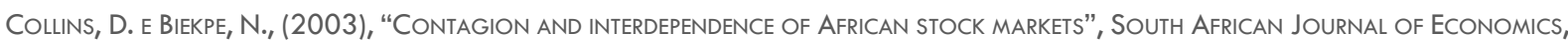
71, 1: 181-194.

Eichengreen, B. e Rose, A., (1998), "Contagious Currency Crisis: Channels of Conveyance." in Changes in Exchange Rates in Rapidly Developing Countries: Theory, Practice, and Policy Issues, Ed. T. Ito e A. Krueger, University of Chicago Press.

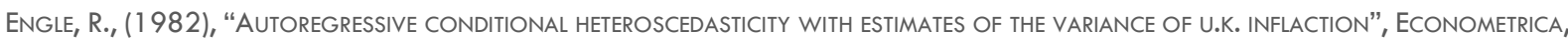
50: 987-1008.

Forbes, K. e Rigobon, R., (2002), "No Contagion, Only Interdependence: Measuring Stock Market Comovements", Journal of FINANCE, 57, PP. 2223-62.

Glick, R. e Rose, K., (1999), "Contagion and Trade: Why Are Currency Crises Regional?", Journal of International Money and FINANCE, 18, PP. 603-617.

Hansen, P., E Lunde, A., (2005), "A forecast comparison of Volatility mOdels: does anything beat a GARCH $(1,1)$ ?", Journal Of APPLIED ECONOMETRICS, 20(7): 873-889.

Horta, P., Mendes, C. e Vieira, I., (2008), "Contagion effects of the US Subprime Crisis on Developed Countries", CefaGe-Ue WORKING PAPER 2008/08.

King, M., E WadhWANI, S., (1990), “Transmission Of Volatility betWeen Stock markets", Review of Financial Studies, 3(1), 5-33.

LiN, W., ENGle, R. e Ito, T., (1994), "Do Bulls and Bears MOVe ACross Borders? International Transmission of Stock Returns and Volatility", ReVIEW Of FinanCIal StUdies, 7, pP. 507-38.

Lin, J. e Treichel, V., (2012), "The Unexpected Global financial Crisis Researching its Root Cause", World Bank. WPS5937. HTTP://WWW-WDS.WORLDBANK.ORG/SERVLET/WDS

CONTENTSERVER/WDSP/IB/2012/01/09/000158349_20120109085942/RenDERED/PDF/ WPS5937. PDF. CONSULTADO EM 2013.

Masson, P., (1999), "Contagion, Monsoonal Effects, Splllovers, and Jumps between Multiple Equilibria." in The Asian Financial Crisis; CAuses, Contagion and Consequences, Ed. P.R. Agenor, M. Miller, D. Vines e A. Weber, Cambridge University Press.

Naoul, K., Khemiri, S. e liouane, N., (2010), "Crises and Financial Contagion: The Subprime Crisis", Journal of Business Studies QUARTERLY, VOL. 2, NO. 1, PP. 15-28.

Nelson, D., (1991), “Conditional Heteroskedasticity in Asset Returns: A New Approach”, EConometrica, 59, 2, 347 - 370. 


\section{Eqitania \\ s c i e $\Omega$ c i a}

Park, Y. e Song, C., (2000), "Financial Contagion in the East Asian Crisis: With Special Reference to the Republic of Korea", In: Claessens, S. and Forbes, K. (Eds.), International Financial Contagion, Massachusetts: KluWer Academic Publishers.

Pestana, M. e Gageiro, J., (2000), AnÁlise de Dados para CiênCias Socials: A complementaridade do SPSS, 2ª edição, Edições Sílabo, LISBOA.

Pindyck, R. e Rotemberg, J., (1990), “The Excess Co-Movement Of Commodity Prices.” The EConomic Journal 100, pp. $1173-89$.

Toussaint, E., (2008), “The US Subprime Crisis Goes Global”, In Counterpunch, Weekend Edition, January 12/13.

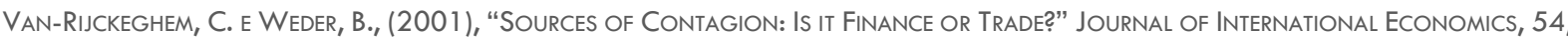
PP. 293-300. 